THE ASTROPHYSICAL JOURNAL SUPPLEMENT SERIES, 90:817-821, 1994 February

c) 1994. The American Astronomical Society. All rights reserved. Printed in U.S.A.

\title{
THE SPECTRAL TURNOVER OF THE "FILLED-CENTER" SUPERNOVA REMNANT 3C 58: IMPLICATIONS FOR WHEN ACCELERATION OCCURS
}

\author{
D. A. GREEN \\ Mullard Radio Astronomy Observatory, Cavendish Laboratory, Madingley Road, Cambridge, CB3 OHE, UK \\ Received 1993 January 28; accepted 1993 April 6
}

\begin{abstract}
IRAS observations the "filled-center" supernova remnant 3C 58 are used to derived upper limits of $0.4,0.4$, 0.8 , and $1.5 \mathrm{Jy}$ for its infrared flux density at $12,25,60$, and $100 \mu \mathrm{m}$, respectively. These values imply a break in the spectrum of $3 \mathrm{C} 58$ above a few tens of gigahertz-two orders of magnitude below the break in the spectrum of the Crab Nebula - with a change of spectral index across the break for 3C 58 of more than 0.5 . This is similar to previous results for another "filled-center" remnant, G74.9+1.2, from radio observations alone. This implies that their emission is not dominated by a constant injection rate of particles with a power-law distribution. Either the break is due to synchrotron losses in the past, with little particle injection in recent times or it is intrinsic to the acceleration mechanism at work (presumably from a central neutron star).
\end{abstract}

Subject headings: acceleration of particles - ISM: individual (3C 58) - radiation mechanisms: nonthermal - supernova remnants

\section{INTRODUCTION}

Most supernova remnants (SNRs) have a limb-brightened "shell" structures at radio wavelengths, with radio spectral indices, $\alpha$ (here defined in the sense $S_{\nu} \propto \nu^{-\alpha}$ ), between $\approx 0.3$ and $\approx 0.8$ (e.g., Scheuer 1984; Green 1988, 1991). However, there are growing classes of SNRs that are either centrally brightened at radio and other wavelengths (e.g., Weiler 1983; Seward 1989), or are "shells" which contain a center-brightened component-these are "filled-center" and "composite" SNRs, respectively. The Crab Nebula is the prototype "filled-center" SNR and is well known to be powered by a central pulsar. "Filled-center" remnants-and the centrally brightened components of "composite" remnants-have radio spectra considerably flatter than those of "shell" remnants, with $\alpha$ between $\approx 0.0$ and $\approx 0.3$. This difference in spectra is due to quite different processes at work within the different classes of remnants: namely, the acceleration in the shocks of "shells" and compared with the production of relativistic particles by the central sources of "filled-center" remnants. The mechanism at work for the central sources of "filled-center" remnants is, however, not well understood. From energy considerations, a break in the synchrotron spectrum of a "filled-center" remnant is expected, and the position of the break should provide a valuable constraint on theories for such emission. In the case of the Crab Nebula the spectral break is at $\approx 10^{4} \mathrm{GHz}$ (Marsden et al. 1984; see also Baldwin 1971) with a change of spectral index of $\approx 0.5$, consistent with synchrotron losses. The reported breaks in the spectra of other "filled-center" SNRs, most of which are thought to be much older than the Crab Nebula, at frequencies between $\approx 10$ and $\approx 100 \mathrm{GHz}$ (Reich, Fürst, \& Sofue 1984; Morsi \& Reich 1987; Salter et al. 1989). In one case, G74.9+1.2, the change of spectral index across the break is reported to be greater than 0.5-this is larger than is expected for synchrotron aging with constant energy injection-although there are uncertainties in the observed flux densities and the small range of frequencies observed above the break frequency.

$3 \mathrm{C} 58(=\mathrm{G} 130.7+3.1)$ is the second best-studied "filledcenter" SNR in the Galaxy (e.g., Wilson \& Weiler 1976; Becker, Helfand, \& Szymkowiak 1982; Green 1986; Reynolds \& Aller 1988). Becker et al.'s X-ray observations provide indirect evidence for a compact source within the remnant, presumably a central power source which leads to the centrally brightened structure of $3 \mathrm{C} 58$ at radio and X-ray wavelengths. Deep radio searches for a central pulsar by Frail \& Moffett (1993) have been unsuccessful. 3C 58 is the remnant of the $\mathrm{SN}$ of A.D. 1181, so is similar in age to the Crab Nebula (the $\mathrm{SN}$ of A.D. 1054). The distances to these two remnants are also similar, but the Crab Nebula is physically smaller, by a factor of $\sim 2$, and is much brighter at all wavelengths (e.g., Green \& Gull 1982; Green 1986), as was its parent SN. Observations at $84.2 \mathrm{GHz}$ by Salter et al. give an integrated flux density of $15.0 \pm 2.0 \mathrm{Jy}$ for $3 \mathrm{C} 58$, which lies just below the spectrum extrapolated from lower radio frequencies, indicating a possible spectral break at several tens of gigahertz. The existence of this possible break is strengthened by upper limits for the infrared flux density by Arendt (1989).

Here I derive strong upper limits on the infrared flux densities are obtained from images made from the IRAS data. For a spectral break at $\approx 50 \mathrm{GHz}$, these limits imply a change in spectral index of more than 0.5 across the break, and provides useful constraints on the time scales or intrinsic spectrum of the mechanism at work, some aspects of which are discussed in Green \& Scheuer (1992). Such breaks may occur at lower frequencies for older "filled-center" SNRs, and this possibility is discussed briefly for the case of DA 495 .

\section{RESULTS}

IRAS Calibrated Raw Detector Data (CRDD) in the direction of $3 \mathrm{C} 58$ were obtained from the IRAS Post Mission Anal- 
ysis Facility staff of the Rutherford Appleton Laboratory in 1991 April. These data consisted of 39 scans in all four IRAS bands taken within $\approx 1^{\circ}$ of $3 \mathrm{C} 58$ (at $02^{\mathrm{h}} 02^{\mathrm{m}} 00^{\mathrm{s}},+64^{\circ} 36^{\prime} 00^{\prime \prime}$, B 1950.0). Thirty-seven of these scans were $\sim 2^{\circ}$ in length, and two were shorter; these latter were excluded from the processing to avoid endeffect problems. The CRDD data were processed using standard STARLINK programs, as outlined by Parsons, Richards, \& Giaretta (1989). The individual scans were "de-striped," with linear gradients and offsets in the detector responses removed, and these were used to produce images $80 \times 80 \operatorname{arcmin}^{2}$ in extent centered on $3 \mathrm{C} 58$.

Figure 1 shows the part of images at the four IRAS wavebands centered on $3 \mathrm{C} 58$. The beam shapes for the observations are approximately rectangular, and orthogonal to the scan directions- which were generally northwest to southeast -as is particularly evident at the shorter wavelengths because of the presence of relatively bright stellar sources in the field. At the longer wavelengths, especially $100 \mu \mathrm{m}$, extended emission dominates the field. These images reveal no obvious emission from 3C 58 itself, although at $60 \mu \mathrm{m}$ there is a faint local maximum near to its position.

In order to place limits on the flux density of $3 \mathrm{C} 58$ at infra-
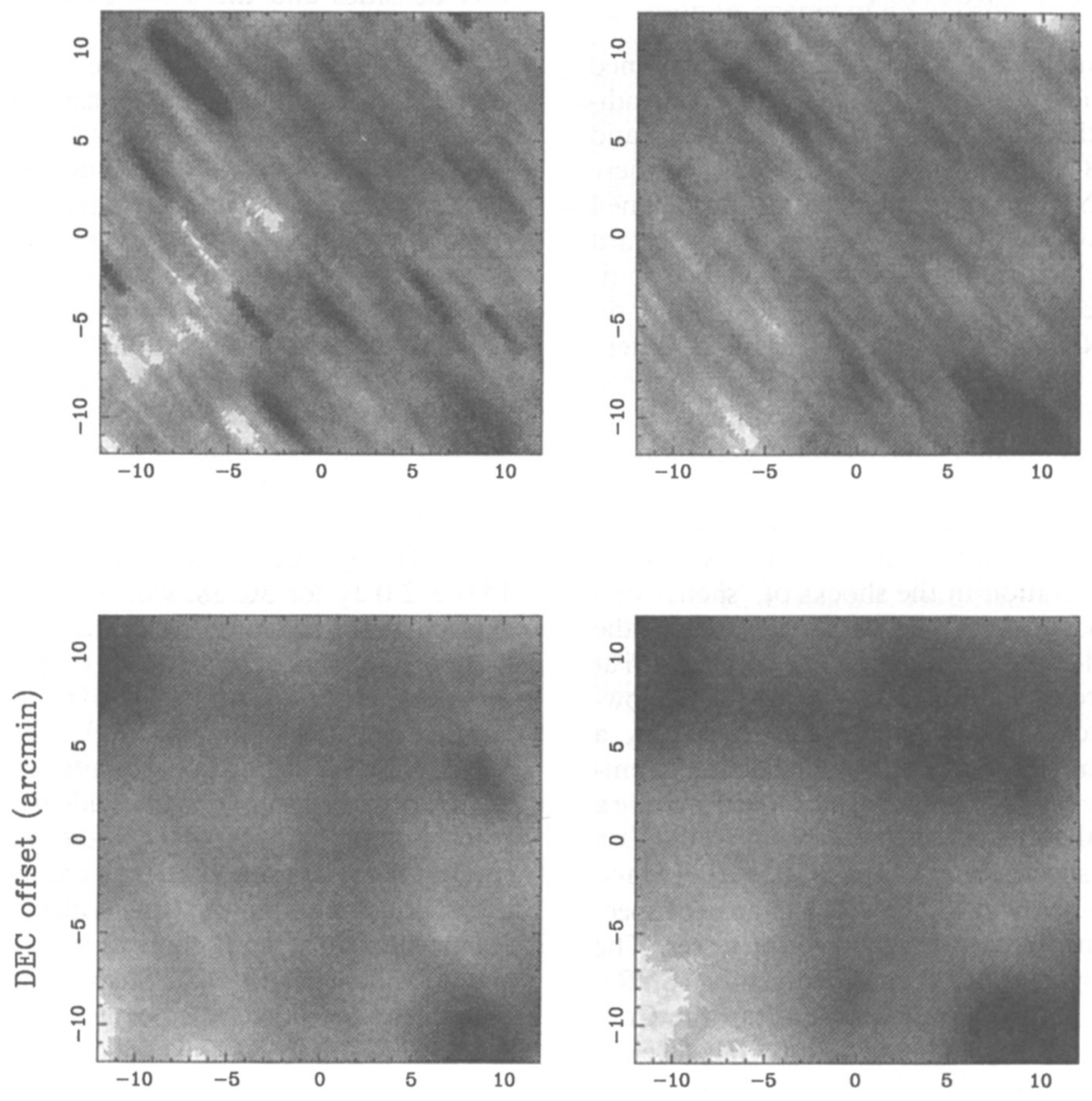

red wavelengths, a simple model of $3 \mathrm{C} 58$ based on radio observations (Green 1986) was constructed from several overlapping Gaussians. This was scaled in intensity by increasing factors and added to the observed infrared images of $3 \mathrm{C} 58$ until the peak of the model emission could be distinguished on gray-scale images at the position of $3 \mathrm{C} 58$. This procedure did not take into account the actual beam of the IRAS images, nor any possible differences in the structure of infrared emission from the radio emission on which the model was based. There may be a decrease in the angular size of emission at increasing frequencies. If the infrared emission is smaller in extent than the model, then the upper limits derived here will be lower, as the model is recognized from its peak. The infrared emission is expected to be smooth, and large compared with the beam size of the observations, so that the lack of convolution of the model with the appropriate beam shape is not very important.

As the identification of the model emission is subjective, the upper limits to the infrared flux density of $3 \mathrm{C} 58$ given here are twice the flux densities at which the model could readily be distinguished when superposed on the observed emission (see Green \& Scheuer 1992). The corresponding upper limits-for infrared emission comparable in structure to the observed ra-

RA offset (arcmin)

Fig. 1.-Images of infrared emission toward $3 \mathrm{C} 58$ from IRAS observations. Coordinates are relative to $3 \mathrm{C} 58$ at $02^{\mathrm{h}} 02^{\mathrm{m}} 00^{\mathrm{s}}, 64^{\mathrm{o}} 36^{\prime} 00^{\prime \prime}(\mathrm{B} 1950.0)$. The gray-scale ranges, white to black, are $(a)-0.1$ to $+0.7 \mathrm{MJy} \mathrm{sr}^{-1}$ for the $12 \mu \mathrm{m}$ image, $(b)-0.1$ to $+0.7 \mathrm{MJy} \mathrm{sr}^{-1}$ for the $25-\mu \mathrm{m}$ image, $(c)-0.3$ to $2.5 \mathrm{MJy} \mathrm{sr}{ }^{-1}$ for the $60 \mu \mathrm{m}$ image, $(d)-0.6$ to $6.0 \mathrm{MJy} \mathrm{sr}^{-1}$ for the $100 \mu \mathrm{m}$ image. $3 \mathrm{C} 58$ is $\approx 9 \times 5 \operatorname{arcmin}^{2}$ in extent, elongated east-west. 
dio emission, as discussed above-are $0.4,0.4,0.8$, and $1.5 \mathrm{Jy}$ at $12,25,60$, and $100 \mu \mathrm{m}$, respectively. These limits are between $\approx 3$ and $\approx 10$ times lower than the previous published limits (Arendt 1989) available from IRAS "Skyflux" plates and co-added data.

Figure 2 shows the radio to infrared spectrum of $3 \mathrm{C} 58$, including the upper limits derived above. The radio spectrum of $3 \mathrm{C} 58$, up to $\approx 32 \mathrm{GHz}$ (see Morsi \& Reich), is well fitted with a spectral index of $0.09 \pm 0.02$ (Green 1986), with indications of a spectral break below $84.2 \mathrm{GHz}$, as Salter et al.'s flux density lies below the spectrum extrapolated from lower frequencies. Thus, the infrared upper limits and radio spectrum of $3 \mathrm{C} 58$ require a spectral break between $\approx 30$ and $\approx 85 \mathrm{GHz}$. For a spectral break in this region, near $50 \mathrm{GHz}$, then the change in spectral index across the break is larger than 0.5 .

A break in the spectrum of $3 \mathrm{C} 58$ at a lower frequency than that of the Crab Nebula $\left(\approx 10^{4} \mathrm{GHz}\right.$ ) is consistent with the relatively low $\mathrm{X}$-ray to radio luminosity of $3 \mathrm{C} 58$ compared with that of the Crab Nebula (Becker et al. 1982; Davelaar, Smith, \& Becker 1986; see also the discussion by Salter et al.).

\section{DISCUSSION}

The radio-infrared spectrum for 3 C 58 raises two problems. First, the break frequency of $\approx 50 \mathrm{GHz}$ is very low for a source less than 1000 years old. For an equipartition field of $\approx 10^{-8} T$, standard synchrotron theory predicts a cutoff frequency of a few times $10^{6} \mathrm{GHz}$, which is many orders of magnitude above the observed break frequency. Second, the change of spectral index is larger than the value of 0.5 predicted by standard Kardashev (1962) theory for a source with continuous injection of particles. In both these respects 3 C 58 is not alone. Morsi \& Reich present observations of the "filled-center" SNR G74.9+1.2 that show that it has a spectral break between 11 and $32 \mathrm{GHz}$, with a change of spectral index of at least 0.8 . In this section I discuss briefly how to explain the observed spectral break in $3 \mathrm{C} 58$ in terms of modifications to synchrotron

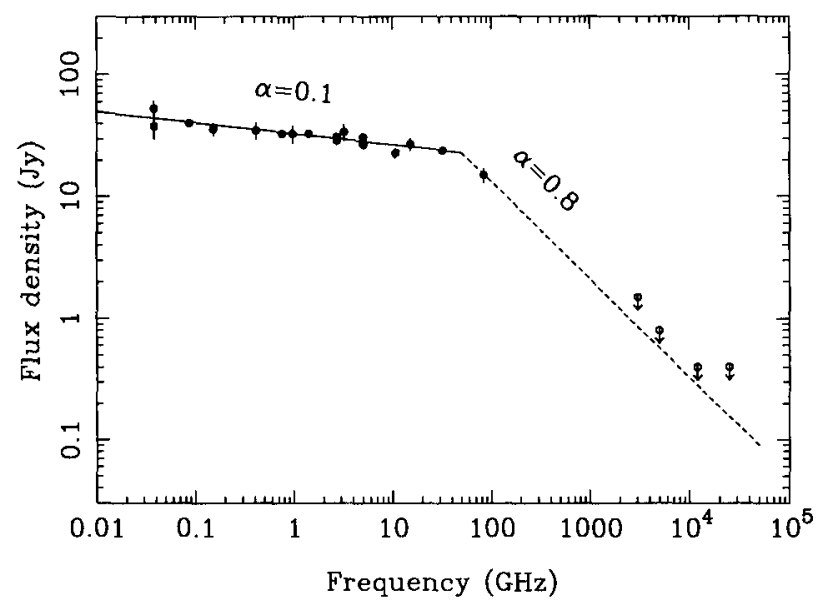

FIG. 2.- The radio to infrared spectrum of 3 C 58 . The radio flux densities are those presented by Green (1986), with additional values: $37.9 \mathrm{Jy}$ (with an estimated uncertainty of $8 \mathrm{Jy}$ ) at $38 \mathrm{MHz}$ (Rees 1990); $24.2 \pm 1.2$ $\mathrm{Jy}$ at $32 \mathrm{GHz}$ (Morsi \& Reich 1987); and $15 \pm 2 \mathrm{Jy}$ at $84.2 \mathrm{GHz}$ (Salter et al. 1989). For clarity, only the extreme $2.7 \mathrm{GHz}$ flux densities ( $27 \pm 2$ and $31 \pm 2 \mathrm{Jy}$ ) of the three available (see Green 1986) are plotted. loss models, or something intrinsic to the particle acceleration process. These points, along with question as to how diffusive effects may influence the observed spectrum are discussed in more detail in Green \& Scheuer.

For standard synchrotron theory, the magnetic field in $3 \mathrm{C}$ 58 would have to be at $\sim 4 \times 10^{-7} \mathrm{~T}$ to bring the break frequency down to $\approx 50 \mathrm{GHz}$. Such a large magnetic field filling the present volume of the source would make the magnetic energy much larger than the kinetic energy (assuming an ISM density of a few atoms $\mathrm{cm}^{-3}$ ) and hence magnetic pressure would then accelerate the expansion until kinetic energy once more exceeds magnetic energy. (Larger fields at earlier times pose less of a problem, as they fill a smaller volume.) Another problem is the steep decline of the spectrum above the break frequency. Since the source is expanding, electrons injected at early times suffer adiabatic expansion as well as synchrotron loss, and therefore the transition from spectral index $\alpha$ to the asymptotic value $\alpha+\frac{1}{2}$ is spread out over a larger frequency range than in a static source. This implies we require a drastic reduction in the rate of particle injection, so that the most recently injected electrons do not cause the synchrotron emission to exceed the $I R A S$ limits.

Reynolds \& Chevalier (1984) discuss models for "filledcenter" SNRs in which magnetic fields and relativistic electrons continuously generated close to a central pulsar form an expanding bubble, and with the inclusion synchrotron losses modified by the effects of expansion leads to a spectra with breaks. (Reynolds \& Chevalier's preferred model for 3C 58 has a flux density of $4 \mathrm{Jy}$ at $10 \mu \mathrm{m}$, which is not consistent with the limits derived above.) Here I consider a less sophisticated model of expanding sphere of relativistic particles and magnetic field, with a simple parameterization of the dynamics in order to investigate what modifications from the basic results are likely. For a sphere which had radius $\rho R$ and uniform magnetic field $\beta B$ at time $\tau T$, where $R, B$ are the radius and magnetic field at age $T=810 \mathrm{a}$, it is possible to calculate the expected synchrotron spectrum for this source (as has been done since Pacini \& Salvati [1973] for SNRs, and by others for radio galaxies, to predict model spectra). Figure 3 shows model synchrotron spectra for $3 \mathrm{C} 58$ based on an intrinsically flat injection spectrum, with different forms of injection rates and magnetic field evolution with $\rho=\tau$ (i.e., constant expansion) and $\beta=\tau^{-b}$. The models are based on a magnetic field of $3.7 \times$ $10^{-7} T$ at present that would give a break frequency of $50 \mathrm{GHz}$ for a 810 year-old static source. Figure $3 b$ shows that moderate modifications of the source history (magnetic field $\propto \tau^{-1}$ or $\tau^{-2}$, injection rate constant or $\propto \tau^{-1}$ ) do little or nothing to improve agreement with observation. Thus the conclusion is that the mean particle injection rate must have fallen sharply in recent times-see Figure $3 a$.

Another possible explanation of the observed spectrum is simply to say the injected distribution had the appropriate shape, either by energy-dependent diffusion or some mechanism, the details of which are not known. As discussed by Green \& Scheuer, if the acceleration mechanism has the break at an energy when (acceleration time scale) $\sim$ (synchrotron loss time) - which is the case in some models - the magnetic field would have to be very large to have the break frequency near $50 \mathrm{GHz}$. Once again the energy requirement is large compared with the kinetic energy of the remnant, unless the acceler- 

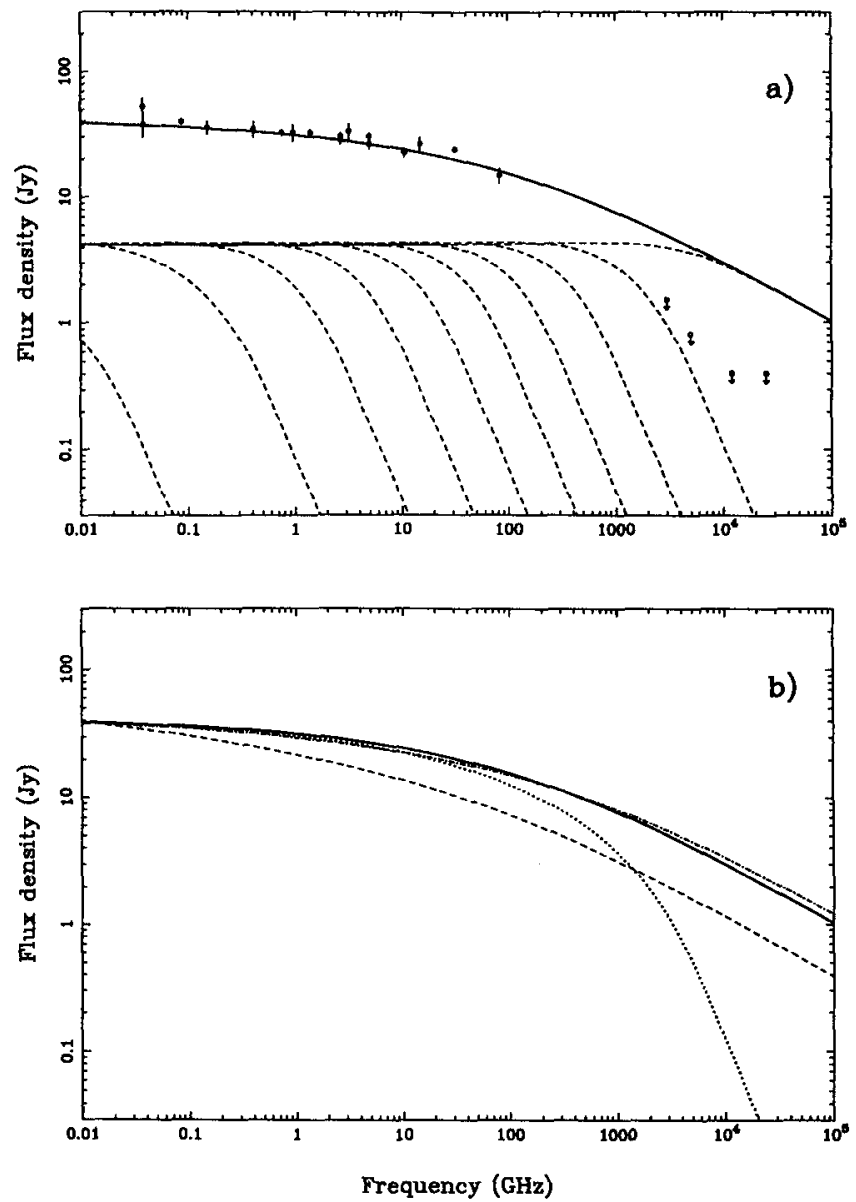

Fig. 3.-Model synchrotron spectra for 3C 58 based on an intrinsically flat injection spectrum, with different forms of injection rates and magnetic field evolution. ( $a$ ) The observed flux densities and limits together with (solid line) a model spectrum based on a uniform injection rate and magnetic field varying inversely with age of the remnant with $b=1$. The dashed lines show the contribution to this total spectrum from each $10 \%$ portion of the remnant's lifetime. (b) The thick solid line is as in $(a)$, the dashed line is for uniform injection but with magnetic field decreasing rapidly with $b=2$, the dashed-dotted line is for nonuniform injection of particles, varying inversely with the age of the remnant, with $b=1$, and the dotted line is for uniform injection with an abrupt cutoff in injection when the remnant was $90 \%$ of its present age. All models are based on uniform expansion and have been normalized to the low-frequency radio observations.

ation takes place in regions of very strong magnetic field close to the central source. In that case, conflict with observation would arise, as a compact central source would be expected to radiate a substantial fraction of the energy of the electrons which are responsible for the synchrotron spectrum near the break frequency. However, no such central source is observed in any frequency range.

\section{CONCLUSIONS}

Analysis of IRASCalibrated Raw Detector Data yields significant upper limits to the flux density of the SNR 3C 58 in all four IRAS bands, and shows that the spectrum of $3 \mathrm{C} 58$ steepens sharply above $\approx 50 \mathrm{GHz}$. Synchrotron losses could account for the observed spectral break provided that the recent particle acceleration rate was much smaller than that prevailing when the electrons now dominating the radiation near 50 $\mathrm{GHz}$ were injected. A constant rate of injection of a power-law energy distribution is not consistent with the observed spectrum. Why a young, active pulsar might switch off so abruptly requires explanation.

Similar conclusions can also be reached for $\mathrm{G} 74.9+1.2$, which shows a sharp break at a lower frequency, and possibly for G21.5-0.9, which shows a break, but it is not clear how sharp this is. It is interesting that the Crab Nebula, the only "filled-center" remnant with a firmly identified central compact source (its pulsar), is the only "filled-center" remnant known with a radio spectrum that extends to high frequencies without a break. Since the existence of a low-frequency breaks in $3 \mathrm{C} 58, \mathrm{G} 74.9+1.2$ and, possibly, also G21.5-0.9 implies that these SNRs do not now contain active pulsars, then fraction of "filled-center" remnants-i.e., one, the Crab Nebula, out of about half a dozen-provides only a lower limit on the pulsar "beaming factor."

Finally, the existence of breaks in the spectra of "filledcenter" SNRs may resolve nature of the anomalous source DA $495(=\mathrm{G} 65.7+1.2)$. This source has classified as a possible "filled-center" remnant by Landecker \& Caswell (1983). However, it has $\alpha \approx 0.6$, which is typical of "shell" rather than "filled-center" remnants. Velusamy et al. (1989) prefer a thick "shell" interpretation for DA 495, although their observations do not show any clearly defined outer boundary. It is possible to reconcile the "filled-center" interpretation with the "shell"like radio spectrum, provided that there is a break in the radio spectrum at frequencies below most available radio observations (a few hundred megahertz). The distance to DA 495 is not known, but if it is indeed Galactic, then it is likely to be several times larger than the Crab Nebula and 3C 58, and, therefore, several times older. This would not be inconsistent with a spectral break at lower frequencies than that in $3 \mathrm{C} 58$ and may also readily explain the minimum in the radio emission at the center of DA 495 seen by Velusamy et al.

I thank P. Scheuer for collaboration on this work, and T. Landecker of DRAO for kindly reminding me of the anomalous nature of DA 495. D. Parsons of the Rutherford Appleton Laboratory kindly supplied the raw CRDD data from the IRAS archive, and the United Kingdom Science and Engineering Research Council are acknowledged for the award of an Advanced Fellowship.

\section{REFERENCES}

Arendt, R. G. 1989, ApJ, 70, 181

Baldwin, J. E. 1971, in IAU Symp. 46, The Crab Nebula, ed. R. D. Davies, \& F. G. Smith (Dordrecht: Reidel), 22

Becker, R. H., Helfand, D. J., \& Szymkowiak, A. E. 1982, ApJ, 255, 557

Davelaar, J., Smith, A., \& Becker, R. H. 1986, ApJ, 300, L59
Frail, D. A., \& Moffett, D. A. 1993, ApJ, 408, 637

Green, D. A. 1986, MNRAS, 218, 533

1988, in NATO ASI Ser. C, Mathematical and Physical Sciences, Vol. 220 Genesis and Propagation of Cosmic Rays, ed. M. M. Shapiro, \& J. P. Wefel (Dordrecht: Reidel), 205 
Green, D. A. 1991, PASP, 103, 209

Green, D. A., \& Gull, S. F. 1982, Nature, 299, 606

Green, D. A., \& Scheuer, P. A. G., 1992, MNRAS, 258, 833

Jones, F. C. 1970, Phys. Rev. D, 2, 2787

Landecker, T. L., \& Caswell, J. L. 1983, AJ, 88, 1810

Kardashev, N. S. 1962, Soviet Astron. 6, 317

Marsden, P. L., Gillett, F. C., Jennings, R. E., Emerson, J. P., de Jong, T., \& Olnon, F. M. 1984, ApJ, 278, L29

Morsi, H. W., \& Reich, W. 1987, A\&AS, 69, 533

Pacini, F., \& Salvati, M. 1973, ApJ, 186, 249

Parsons, D. C., Richards, P. J., \& Giaretta, D. L. 1989, Starlink User Note 91, Rutherford Appleton Laboratory
Rees, N., 1990, MNRAS, 244, 233

Reich, W., Fürst, E., \& Sofue, Y. 1984, A\&A, 133, L4

Reynolds, S. P., \& Aller, H. D. 1988, ApJ, 327, 845

Reynolds, S. P., \& Chevalier, R. A. 1984, ApJ, 278, 630

Salter, C. J., Reynolds, S. P., Hogg, D. E., Payne, J. M., \& Rhodes, P. J. 1989, ApJ, 338, 171

Scheuer, P. A. G. 1984, Adv. Space Res., 4, 337

Seward, F. D. 1989, Space Sci. Rev., 49, 385

Velusamy, T., Becker, R. H., Goss, W. M., \& Helfand, D. J. 1989, J. Astrophy. Astron. 10, 161

Weiler, K. W. 1983, Observatory, 103, 85

Wilson, A. S., \& Weiler, K. W. 1976, A\&A, 49, 357 